\title{
Amelia Edwards à Karnak
}

L'enthousiasme d'une voyageuse anglaise pour l'Égypte ancienne (1877)

\section{Caroline Lehni}

Traducteur : Caroline Lehni

\section{(2) OpenEdition}

\section{Journals}

Édition électronique

URL : https://journals.openedition.org/rbnu/3378

DOI : $10.4000 /$ rbnu.3378

ISSN : 2679-6104

\section{Éditeur}

Bibliothèque nationale et universitaire de Strasbourg

\section{Édition imprimée}

Date de publication : 1 novembre 2010

Pagination : 6-7

ISSN : 2109-2761

\section{Référence électronique}

Caroline Lehni, «Amelia Edwards à Karnak », La Revue de la BNU [En ligne], 2 | 2010, mis en ligne le 01 novembre 2010, consulté le 08 août 2021. URL : http://journals.openedition.org/rbnu/3378 ; DOI : https://doi.org/10.4000/rbnu.3378

\section{(c) (i) (2)(2)}

La Revue de la BNU est mise à disposition selon les termes de la Licence Creative Commons Attribution - Pas d'Utilisation Commerciale - Partage dans les Mêmes Conditions 4.0 International. 


\section{Amelia Edwards à Karnak : l'enthousiasme d'une voyageuse anglaise pour l'Égypte ancienne (I877)}

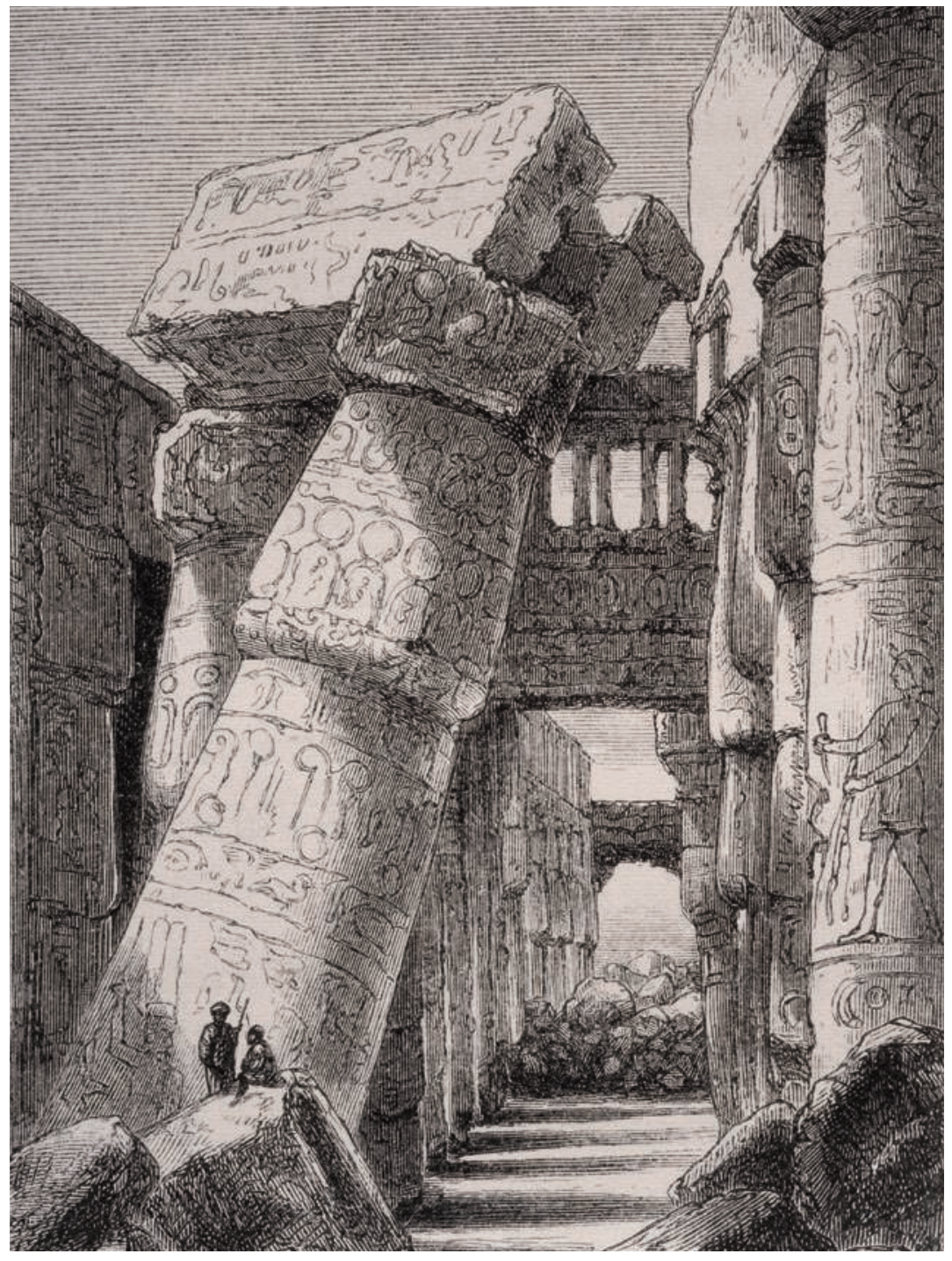

La salle hypostyle de Karnak. Illustration tirée de la première édition de l'ouvrage (Londres : Longmans, Green, \& Co., 1877) 
A ppelée à sa mort «la reine de l'égyptologie ${ }^{1}$ ", Amelia Edwards n'est pas une voyageuse anglaise tout à fait comme les autres. Contrairement aux "Cook tourists ", et aux nombreux sujets britanniques qui remontent le Nil chaque hiver depuis les années 1840, cette romancière passionnée par l'Égypte antique se veut consciencieuse et appliquée : sa croisière sur le Nil durant l'hiver 1873-1874 est un véritable voyage d'étude, qu'elle complète à son retour par des mois de recherches avant de publier, en 1877, A Thousand Miles up the Nile ${ }^{2}$. Si les nombreuses anecdotes de ce récit de voyage portent la marque de son talent d'écrivain populaire, la précision des descriptions de monuments et l'érudition des explications historiques, souvent conformes aux découvertes archéologiques les plus récentes, en font une introduction de qualité à l'art et à l'histoire de l'Égypte ancienne. Les illustrations, tirées des croquis de la voyageuse et gravées sur bois de bout, participent pleinement au double projet de l'ouvrage, qui se veut aussi instructif que divertissant. Avec ce livre, A. Edwards ne se contente pas de publier un énième récit de voyage en Égypte ; elle se met au service de l'égyptologie. Jusqu'à sa mort en 1892, elle continue de se consacrer à l'étude de l'Égypte antique, à la diffusion des connaissances issues de la recherche égyptologique et à la conservation de ce patrimoine en participant notamment à la fondation de l'Egypt Exploration Fund en 1882.

Dans le passage que nous avons choisi de présenter ici pour la première fois en traduction française ${ }^{3}$, Amelia Edwards relate sa découverte du grand temple de Karnak aux côtés de ses compagnons de voyage. À propos de la grande salle hypostyle, elle reprend un topos de la littérature de voyage, celui de l'impossibilité de décrire un lieu trop beau ou trop grand. L'usage de ce topos s'inscrit dans la rhétorique du sublime à laquelle l'auteur a largement recours dans les pages consacrées à Karnak. A. Edwards va cependant plus loin et construit un parallèle rigoureux entre l'impossible description et l'impossible représentation graphique. La présence d'une illustration, sous la forme d'une vignette rectangulaire, atteste néanmoins du statut rhétorique de la double prétérition de ce texte : de même qu'elle finit par livrer une description du lieu, A. Edwards en donne également une représentation graphique. La fonction principale de ce passage est donc de tenter de traduire l'expérience esthétique de la voyageuse sur les lieux. L'évocation sensible va cependant de pair avec des éléments d'information précis. Certes, ils sont parfois exposés au moyen d'une terminologie inadéquate, mais celle-ci a le mérite d'être familière au lectorat bourgeois cultivé auquel l'ouvrage est destiné. À la fin de ce passage, l'auteur va même jusqu'à défendre de façon argumentée la thèse à laquelle elle souscrit concernant un point d'interprétation archéologique. Auteur à succès, voyageuse enthousiaste, Amelia Edwards s'affirme dans ce passage comme une égyptologue amateur éclairée. C'est ce que le célèbre Auguste Mariette avait bien compris à la lecture de $\mathrm{A}$ Thousand Miles up the Nile, lui qui, en juin 1877 , écrivit à l'auteur les lignes suivantes :

Je voudrais que l'Égypte fût visitée tous les ans par des voyageurs comme vous; je voudrais que tous les ans on publiât sur l'Égypte des ouvrages aussi intéressants et aussi consciencieusement étudiés que le vôtre [...] Les erreurs souvent extraordinaires qui ont cours dans le public sur l'Égypte ancienne finiraient par se transformer en notions justes et saines ${ }^{4}$.

« Nous poursuivîmes notre chemin. Laissant à droite un colosse mutilé portant, sur le bras et la poitrine, le cartouche de Ramsès II, nous franchîmes le seuil ombragé et entrâmes dans la célèbre salle hypostyle de Séti Irer.

C'est un lieu sur lequel on a beaucoup écrit et que l'on a souvent peint, mais dont nulle écriture et nulle peinture ne peuvent offrir plus qu'une impression insipide et amoindrie. Le décrire, c'està-dire en donner une image au moyen de mots, voilà qui est impossible. L'échelle en est trop vaste, l'effet produit trop énorme, et le sentiment que l'on a de ses propres limites, de sa petitesse et de son incapacité est trop fort et trop écrasant. C'est un lieu qui impose le silence, qui semble vous vider non seulement de mots, mais aussi d'idées. Et ce n'est pas qu'une première impression. Plus tard dans l'année, lorsque nous redescendîmes le Nil et mouillâmes près des ruines pour y passer de longues journées, je découvris que je ne pouvais toujours pas dire un mot dans la grande salle hypostyle. Certains ont pu mesurer la circonférence de ces énormes colonnes; d'autres encore ont pu grimper çà et là afin de 
trouver des points de vue ou de vérifier l'exactitude des propos de Mariette et Wilkinson ; quant à moi, je n'ai pu que regarder, et rester silencieuse.

Mais regarder, c'est déjà quelque chose, si du moins l'on parvient à se rappeler ce que l'on a vu; et la grande salle de Karnak est photographiée dans un coin sombre de ma mémoire pour aussi longtemps que je conserverai des souvenirs. Je ferme les yeux et je la revois comme si j'y étais, pas toute entière à la fois comme dans une image, mais élément par élément, à la façon dont l'œil prend en compte de grands objets ou survole un large champ de vision. Je me tiens une fois de plus parmi ces colonnes majestueuses qui, d'où qu'on les regarde, forment des avenues rayonnantes. Je les vois, enveloppées par des spirales d'ombre et de larges bandes de lumière. Je les vois, couvertes de bas-reliefs et de peintures montrant des dieux et des rois, des noms royaux blasonnés, des autels sacrificiels, des silhouettes d'animaux sacrés et des emblèmes de la sagesse et de la vérité. Les fûts de ces colonnes sont énormes. Je me tiens au pied de l'une d'elles [...] Six hommes debout, leurs bras tendus de chaque côté, se touchant du bout des doigts pourraient à peine en faire le tour [...] Le chapiteau, qui me surplombe de si haut, paraît avoir été placé là pour supporter les cieux. Il est sculpté de façon à ressembler à une fleur de lotus épanouie, brillant des feux de ses couleurs immortelles - des couleurs toujours vives bien qu'ayant été appliquées par des mains devenues poussière il y a plus de trois mille ans. Il faudrait non pas six, mais une douzaine d'hommes pour mesurer la courbe des lèvres de ce lis extraordinaire.

Telle est l'apparence des douze colonnes centrales. Les autres (au nombre de cent vingt-deux) sont elles aussi gigantesques, mais plus petites. Du plafond qu'elles supportaient autrefois il ne reste que les poutres. Ces poutres sont des pierres, d'immenses monolithes gravés et peints, qui enjambent l'espace entre les piliers et projettent sur le sol un motif de bandes d'ombre.

[...] Il est difficile d'imaginer cette salle couverte de son plafond, sans ouverture sur le ciel. Elle semble parfaite ainsi et l'on se dit, au fond, qu'il ne devrait rien y avoir entre de telles colonnes et les profondeurs bleues infinies du ciel.

Toutefois, la grande avenue centrale était autrefois éclairée grâce à une double rangée de fenêtres à claire-voie, dont quelques-unes subsistent encore.
Certains auteurs ont suggéré que ces fenêtres pourraient avoir été vitrées, mais cela semble improbable pour deux raisons. D'abord, parce qu'un ou deux des encadrements de fenêtres ont conservé le grillage en pierre qui semble bien avoir rempli les fonctions d'un matériau translucide. Ensuite, parce que nous n'avons aucune preuve que les anciens Egyptiens, qui connaissaient pourtant la technique du soufflage depuis l'époque de Chéops, aient jamais fabriqué du verre en feuilles ou utilisé celui-ci de cette façon dans leurs bâtiments. "

Amelia Edwards, A Thousand Miles up the Nile, London, Routledge, 1888, p. 148-150

Présentation et traduction de Caroline Lehni

\section{Notes}

1 - L. W. Winslow, "Amelia B. Edwards the 'Queen of Egyptology' ", The American Antiquarian, novembre 1892, cité par Billie Melman, Women's Orients : English Women and the Middle East, 1718-1918, London, Macmillan, 1992, p. 255

2 - Amelia Edwards, A Thousand Miles up the Nile, London, Longmans, Green, \& Co., $1877 ; 2^{\mathrm{e}}$ éd. revue et corrigée, London, Routledge, 1888

3 - Plusieurs rééditions de A Thousand Miles up the Nile ont paru ces dernières années, parmi lesquelles une réédition en fac-similé de la deuxième édition (London, Darf, 1993). Il n'existe à ce jour aucune traduction française de l'ouvrage.

4 - Oxford, Somerville College Library, Amelia Edwards Collection, cat. $\mathrm{n}^{\circ}{ }^{121}$, lettre de Mariette Pacha, 13 juin 1877 , f. 1-2 